and asthma are of vaso-motor mechanism and that they depend essentially upon a widespread vaso-constriction compensated wholly or in part by a more localised vaso-dilation. Conformably I have shown that in migraine the pain may be relieved (often completely though temporarily) by the simultaneous applications of heat to the general surface of the trunk and cold to the scalp; and that in asthma the dyspnoea may be relieved (often completely though temporarily) by the application of heat to the general surface of the trunk, combined with the inhalation of cold air. Again, in the same place I have argued that the mechanism of the physiological process of menstruation also depends upon a widespread increase of vaso-constriction or of general arterial tone, compensated at any rate in part by a pelvic, especially a uterine, vaso-dilation; and that the uterine vascular distension so attained results naturally in the menstrual loss. Conformably, I have shown that the loss is conspicuously reduced by hot immersion during menstruation.

Now in all three cases the results-diminution of pain in migraine, of dyspnoea in asthma, ancl of hæmorrhage in menstruation-are attained similarly-namely, by a reversal of the mechanism responsible for the phenomena of the pathological processes and the physiological process respectively. In all three cases such reversal results in diminution of the vascular distension at the site of the production of symptoms. And it follows that the relief of hæmoptysis by a similar combination of heat and cold would be attained by a similar modification of vaso-motor mechanism.

It may be, of course, that the treatment of phthisical hæmoptysis by the thermal means suggested would be inexpedient on account of incidental dangers not here referred to. Having no practical experience of the method, I could not defend it against such a charge. In any case. however, the method would remain a rational one-a remark which cannot, I maintain, be applied to the present widely adopted plan of placing an icebag in contact with some portion of the skin covering the thorax.

\section{THE TREATMENT OF CONSTIPATION BY THE INJECTION OF OLIVE OIL.}

\section{BY GEORGE HERSCHELL, M.D. LOND.}

AlTHOUgh introduced into practice a considerable time ago by Kussmaul and Fleiner, the treatment of constipation by the injection of olive oil appears to have been much neglected in this country. Whilst without question most valuable in obstinate cases of constipation, especially when due to spasmodic contraction of the bowel, we find in the methodical use of oil injections one of the most useful procedures which we have at our command for the treatment of muco-membranous colitis. Fior not only does it to a great extent relieve the constipation which is so often present but it appears to reduce the amount of mucus in a remarkable manner. In fact, in many cases the amelioration amounts practically to a cure.

There are two points to be observed if we wish to be successful with this method. First, that we select cases which are suitable and, secondly, that the injections are given in a proper manner. If we prescribe the injection of olive oil as a matter of routine in all the cases of constipation which come to us for treatment we shall certainly be disappointed and discredit a very valuable method. For instance, we can hardly expect to do any permanent good to cases which depend upon improper food, or hard drinking water, or are the result of pyloric stenosis or gastric myasthenia. In like manner we cannot restore defective sensibility to the interior of the rectum by injections of any kind. But, per contra, we shall invariably obtain results of a very gratifying nature in (1) eases depending upon chronic colitis; (2) constipation associated with spasm of the bowel such as we so frequently find in neurasthenia; and (3) we may use this method with advantage to secure a daily action of the bowels in atony of the intestines whilst the affection is being treated by electrical methods. In these last cases the first week or two are most trying to both physician and patient as all purgatives have been abandoned and the treatment has not yet had time to restore sufficient tone to secure a daily relief In these circurnstances oil injections render us invaluable aid and if properly given are usually sufficient to keep the patient comfortable.

The method consists in the injection of from three to ten ounces of warm olive oil into the rectum at bedtime. This is retained during the night and usually results in an evacuation after breakfast on the following day. If the oil is introduced slowly at a low pressure by the force of gravity it should not produce an immediate call to stool and the patient should have no difficulty in retaining it for some hours. The Higginson syringe is the worst possible apparatus for injecting the oil as the average patient cannot manipulate it in such a manner as to produce a gentle and equable flow, the pressure under which the oil enters the body is far too high, and it cannot be easily used single-handed whilst the patient is in the recumbent position. From this it follows that as it is usually impossible for the physician or a trained nurse to give the daily injection we must provide the patient with an apparatus which can easily be used unaided and which from its construction compels a proper technique.

The apparatus which I have devised to meet these indications is very simple, consisting of a glass funnel (A) of a large relative capacity to its height, provided with a metal loop (B) by which it can be suspended at a convenient height above the bed upon which the patient lies. It is fitted with

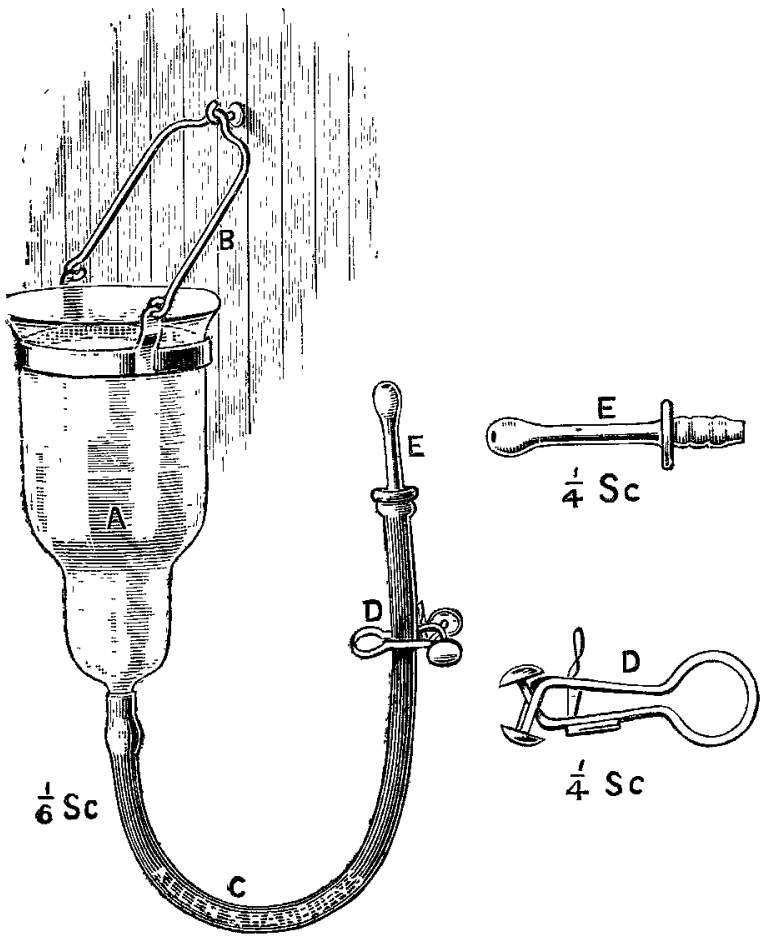

about 27 inches of rubber tube (c) of large calibre, and terminates in a nozzle (E) of special construction. This latter has a large bore to allow the ready passage of the oil which invariably clogs the nozzles supplied with ordinary enema apparatus, and has the end of the bore well rounded so that even when roughly and unskilfully used it is impossible to damage the mucous membrane of the rectum, and from its shape is self-retaining. For durability and cleanliness it has been constructed of aluminium. The outflow of oil is controlled by a spring clip shown at (D) which is so contrived that when opened it will remain so until a catch has been released.

The injection of the oil can be readily performed by the patient without assistance. The measured quantity of oil is heated by standing the beaker containing it in a basin of hot water, it is emptied into the funnel taking care that the clip is closed, the funnel is hung on a nail driven into the wall above the bed, the patient lies on his back directly under it, places a pillow under his hips, introduces the nozzle, presses the clip, and waits until the funnel is empty. For the first few times it may be as well to apply a pad of wool to the perineum to absorb any oil which may not be retained; afterwards this precaution will be superfluous. It is best to commence with five or six ounces and to reduce the dose daily until the smallest amount which will produce an action of the bowels is found. If five ounces is not sufficient it may be raised to ten but beyond this it is not advisable to go and in cases where this 
is insufficient the oil may for a few days be supplemented with a small water injection before breakfast. In any case after a few days it will probably be found that a few ounces of oil alone at bedtime will produce a daily evacuation. When this stage has been arrived at this dose can be given nightly for two or three weeks. The effect may then be tried of using the oil on alternate nights. It will probably be found that the bowels will be opened on the days following the intermission and as the case progresses the action of the oil will extend over a longer and longer time until it will be found that each injection will be followed by several daily stools. When this period arrives the injection should be ordered to be taken only on the evening of a day upon which an action has not taken place.

Har'ey-street, $\mathbf{w}$.

\section{A RETROPHARYNGEAL BLOOD CYST IN A CASE OF PURPURA H EMORRHAGICA.}

\section{By P. STANLEY BLAKER, M.R.C.S. ENG.,} L.R.C.P. LOND.,

LATE RESIDENT MEDICAL OFFICER, EAST LONDIN HOSPITAL FOR CHILDREN, SHADWELL, E.

THE patient was a very well-nourished female child eight months old. She was taken to the Children's Hospital by her mother on June 6th, 1902, with the following history. The child had been ill for six days previously. The mother noticed on June 1st that the patient was not as bright and happy as usual. On the next day there was an attack of epistaxis; this lasted for some time and recurred off and on until the time of admission into hospital. On the same day that the epistaxis began the mother noticed that the child apparently experienced some difficulty in swallowing her milk. On June 3rd a rash in the form of dark red spots appeared on various parts of the body, especially about the region of the knees, and in addition to the dysphagia there was some difficulty in breathing. Fresh spots had developed by the 4th "and the old ones had turned of a purplish colour." On the 5th " all the symptoms had become more aggravated and the child's condition was serious, fresh spots had appeared and the dysphagia and dyspnoes were both more marked." On the 6th the patient was taken to the hospital and admitted. During this time she had been sick, occasionally bringing up blood; the bowels were rather relaxed and the motions were of a dark colour. The previous history showed that she had never been ill before; she had been brought up on the breast. The father and mother were both very healthy; the father's sister died in childbirth; the mother's father was said to have suffered from epistaxis. The mother lost one child from diphtheria and there was one other child living who was quite well. There were no miscarriages.

On admission the child was pale, with the eyes rather sunken and the lips somewhat cyanosed. The respirations were rapid, shallow, and stertorous; there was some recession of the lower ribs, with sucking in of the intercostal spaces during inspiration. From the anterior nares there was a copious discharge of mucus, not blood-stained. The anterior fontanelle was still open, there were two lower incisor teeth, and the gums were quite healthy. There were no very obvious signs of rickets; no tenderness or swelling over any of the bones was present. Subcutaneous hæmorrhagic spots were scattered all over the body, including the trunk, extremities, and face, and especially about the front of the knees. Some of the bigger spots were of about the size of a split pea and were very dark in colour. In addition there were several small bruises over the lower third of both thighs and on both arms. On the left side of the neck behind the posterior border of the sterno-mastoid muscle was a nonfluctuating swelling of glandular origin. A large quantity of mucus in the mouth obscured the pharynx and surrounding structures from view, but a digital exploration revealed a distinct tense elastic swelling of about the size of half a walnut situated on the posterior pharyngeal wall more to the left side. This exploration was followed by bleeding from the nose. The action of the heart was rather rapid but otherwise the sounds were quite natural. 'The lungs were free from any signs of trouble beyond a few rhonchi heard here and there. The abdomen was natural and the liver and spleen could noither of them be felt. There was no blood in the urine as far as could be seen from the wet napkins.

On first sceing the child diphtheria was suspected and a smear was made on a cover-slip from the nasal discharge and stained, but it was found to be free from diphtheritic organisms. It was after this that the digital exploration of the throat was made and the swelling detected. Of course, an acute retropharyngeal abscess was diagnosed and as there was a good deal of obstruction to the breathing it was decided to open the abscess immediately by an incision in the mouth. The patient was taken into the operating theatre and without an anæsthetic the swelling was incised, the knife being guarded and directed along the left index finger. During the operation the head was thrown well over the edge of the table and turned over immediately the incision was made. Dark-coloured blood escaped from the mouth but there were no signs of pus. There was very free bleeding after the operation but the breathing was instantly relieved. The hæmorrhage continued without ceasing till the patient died. A good deal of it came through the nose and large quantities were vomited every now and then. An alum spray ( 30 grains to one ounce) was frequently used; glycerine and tannic acid (20 grains to one ounce) was painted over the parts, likewise tinctura ferri perchloridi ( 1 in 4 of water); sponges soaked in iced water were applied to the pharynx and finally adrenalin solution was painted over the part; internally calcium chloride (five grains) was given, to be repeated every three hours. None of these remedies were effectual in checking the bleeding. Death took place five hours after the operation.

Nccropsy.-The post-mortem examination was made on the following afternoon. The body was well nourished and was very pale. Rigor mortis was not present. There were many small bruises and subcutaneous hæmorrhagic spots as above described. The gums and tonoue were quite natural. In the posterior pharyngeal wall, more to the left side and on a level with the epiglottis, was the cavity of a bloor cyst of about the size of a large cherry which had been opened by a small incision. Its walls were thick and wore composed of the submucous tissues. The cesophagus, larynx, trachea, and bronchi were all natural. The glands in the left side of the neck were enlarged. As regards the heart there were small petechial hæmorrhages into the epicariium but there were none into the endocardium. On the surface of the lungs there were a few petechize and on section several red congested areas were scattered throughout. The thoracic glands were all natural. The liver and spleen were normal. The kidneys and suprarenal bodies were likewise normal. The stomach contained altered blood and at the cardiac end there were many large submucous hæmorrbages. 'The whole length of the intestinal canal contained dark altered blood and in the lower half of the small intestine and colon there were many small submucous hæmorrhages. The solitary follicles and Peyer's patches were distinctly swollen. There was well-marked craniotabes of the back of the skull and there were also some small hæmorrhages under the pericranium and also beneath the dura mater, particularly in the posterior fossa. The brain and meninges were quite normal. A tube of broth was inoculated from a freshly cut section of the spleen but it remained perfectly sterile.

The case is extremely interesting from the point of view of diagnosis and treatment. With regard to the former the diagnosis must first be made of the general disease and secondly of the swelling in the pharynx. The onset in this case was rather too quick to fit in with the diagnosis of scurvy-rickets nor were there any of the characteristio tenderness and subperiosteal swellings of any of the long bones. The gums were quite healthy and the fact that the infant had been brought up on the breast was all against that theory. Hæmophilia in the female child is an extremely rare condition (a case of the kind was recently recorded by $\mathrm{me}^{1}$ ) nor was there anything in the family history to support that view. Lastly, the case might have been taken for one of those somewhat rare examples of suprarenal apoplexy associated with a general hæmorrhagic diathesis. My experience of this disease is that it is very acute in its onset and rapidly fatal, being preceded by severe collapse. The necropsy in the present case showed the suprarenal bodies to be perfectly healthy. Purpura hæmorrhagica is a condition that is fairly frequently met with in children, 